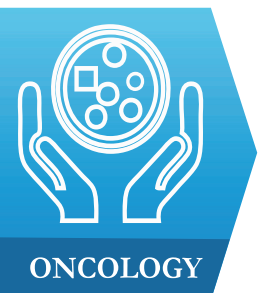

1) Medical Oncology Department, Iuliu Hatieganu University of Medicine and Pharmacy, Cluj-Napoca, Romania

2) $2^{\text {nd }}$ Pediatrics Department, Iuliu Hatieganu University of Medicine and Pharmacy, Cluj-Napoca, Romania
DOI: $10.15386 /$ cjmed-1104

Manuscript received: 17.07 .2018 Received in revised form: 28.09 .2018 Accepted: 12.10.2018

Address for correspondence: surgenel@yahoo.com

\title{
Colorectal cancer: evolution of screening strategies
}

Daniel Sur ${ }^{1}$, Marius Colceriu ${ }^{2}$, Genel Sur ${ }^{2}$, Emanuela Floca ${ }^{2}$, Loredana Dascal $^{2}$, Alexandru Irimie ${ }^{1}$

\begin{abstract}
Background and aim. Colorectal cancer is considered to be a major public health problem. It is the third most frequent cancer at a global level and also the fourth most frequent cause of death. Previous scientific findings have proved that a significant percentage of colorectal cancer deaths are due to the abscence of screening. The aim of this review is to present the evolution of the screening strategies by using the most recommended and recent colorectal cancer screening guidelines.

Methods. A systematic literature search on the scientific databases was performed, identifying some of the most important colorectal cancer screening guidelines publications.

Results. The most recent guidelines of American Cancer Society (2018) recommend that adults aged 45 years and older with an average risk of colorectal cancer should undergo regular screening. All the guidelines have considered fecal occult blood testing (annual or biennial), fecal immunochemical test (annual), flexible sigmoidoscopy (every 5 years) and colonoscopy (every 10 years) as the most preferred screening options. However, there are discrepancies with regards to which tests should be preferred for screening.

Conclusion. Increased compliance with colorectal cancer screening recommendations has the potential to improve patients' health and to reduce colorectal cancer morbidity and mortality rates. It is important for health care providers to have an understanding of the risk factors for colorectal cancer and various stages of disease development in order to recommend appropriate screening strategies.
\end{abstract}

Keywords: colorectal cancer, screening, guidelines

\section{Problem statement}

Colorectal cancer (CRC) is considered to be a major public health problem [1]. It is the third most frequent cancer worldwide and also the fourth most frequent cause of death [2]. According to the World Health Organization (WHO), $\mathrm{CRC}$ is the second most common cancer diagnosed among women and the third most common cancer diagnosed among men [3]. At the European level, it is the most common cause of cancer death following lung cancer [4]. At a national level, in Romania the incidence of CRC is increasing [5].

There is a wide variation of CRC rates among specific geographic areas due to different exposure to risk factors, screening and also access to appropriate treatment services. It is considered to be a disease of the developed world, but the rates of the new cases of CRC have been increasing in the developing countries. The global burden is expected to increase due to the growth and aging of the population and because of behaviors and lifestyle [6]. It is estimated that by 2030 the global burden of CRC will increase by $60 \%$ to more than 2.2 million new cases and around 1.1 million deaths [7].

CRC is considered to be a nearly ideal disease for screening because it can be identified and definitively treated, preventing both the mortality and morbidity associated with the clinical course of this important disease [8].

Previous studies have demonstrated a broad variation in preferences for the CRC screening tests, as tests differ regarding benefit and potential harm. Guidelines tend to underline the role of individual preferences in the selection of a 
screening test, as they have been found to influence uptake in a CRC-screening programme. There is insufficient evidence to recommend one screening test over another [9]. Patients will have a preference for one type of screening test over other tests if provided sufficient information regarding these tests [10]. The adherence rate to CRC screening is generally more important than the screening strategy that is being used [8].

There are different screening tests for the detection of CRC, each of which has advantages and limitations. The aim of this review is to present the evolution of the CRC screening strategies by using the most recommended CRC screening guidelines.

\section{Individuals at risk for CRC}

Several factors are associated with an increased risk for the disease. Some risk factors are modifiable, such as obesity, diet, lack of physical activity, smoking and alcohol consumption. Some protective factors are: higher intake of dietary fiber, green leafy vegetables, calcium and folate [11].

Other risk factors cannot be modified, such as racial and ethnic backgrounds, a personal or familial history of colorectal polyps or CRC, Lynch syndrome, a personal history of inflammatory bowel disease, and the presence of type 2 diabetes [11].

Advancement in age increases the risk of developing CRC. It is estimated that $90 \%$ of the CRC cases occur in people aged 50 or older [12]. The incidence rates of CRC are estimated to be 50 times higher in individuals aged 60 to 79 compared with those younger than 40 years. However, the cases of $\mathrm{CRC}$ have been increasing among younger individuals [13].

High-risk individuals refer to patients with personal or familial history of CRC and adenomas, hereditary CRC syndromes, adenomatous polyposis, and inflammatory bowel disease. These patients should consult with their health care providers to determine when to begin screening and how often to repeat it [11].

\section{CRC screening}

Previous scientific findings have proved that a significant percentage of $\mathrm{CRC}$ deaths are due to absence of screening [14]. Knowledge of preventive methods, improvements in therapeutic and diagnostic procedures, advances in the quality of preoperative assessment and treatment have been proven to contribute to the decrease of the CRC cases [2]. Less invasive technologies have been developed in order to replace the highly invasive colonoscopy [15].

\section{Screening methods for CRC}

Non-invasive tests refer to stool and blood-based tests along with radiologic tests [16]. Available screening methods for CRC refer to fecal occult blood testing (FOBT) that can be guaiac based or immunochemical. It has been proved in randomized control trials that annually FOBT decreases the colorectal mortality rates by approximately $30 \%$. These tests include detecting blood or shredded cell debris by vascularized polyps, adenomas and cancers [3].

Invasive methods of screening refer to the flexible sigmoidoscopy (FS) and colonoscopy which provide direct visualization and identification of a colonic poly or advanced neoplasia with the benefit of getting a pathology specimen [17]. Colonoscopy is considered the gold standard instrument of screening with a high specificity and sensitivity. These tests provide the opportunity to identify, detect and resect neoplasia and precancerous lesions among the entire large bowel and are considered the definitive examination when the other screening tests are positive [18].

Recommended guidelines for CRC screening

Organizations such as American Cancer Society (ACS), United States Multi-Society Task Force on Colorectal Cancer (MSTF), and American College of Radiology (ACR) among with U.S. Preventive Services Task Force (USPSTF), American College of Physicians (ACP), American College of Gastroenterology (ACG) and National Comprehensive Cancer Network (NCCN) have focused on guidelines regarding CRC screening [3].

Guidelines recommend regular screening for CRC and adenomatous polyps in asymptomatic individuals aged 50. However, these guidelines are different with regard to the frequency of screening, to the recommended age when the screening should stop and also with regard to the most frequent and preferred screening methods. For patients at high risk, the recommendations are different with regard to the age at which they should begin screening, the frequency and also the method of screening [10].

A joint guideline described by these three organizations MSTF, ACS, and ACR - 2008 recommends that screening for adenomatous polyps and CRC should start with individuals aged 50 in asymptomatic women and men. For the individuals having CRC risk factors (family history of CRC or polyps, personal history of CRC, personal history of chronic inflammatory bowel disease and family history of hereditary CRC syndrome such as familial adenomatous polyposis or hereditary non-polyposis colon cancer) it is suggested that they should undergo colonoscopy at an early age and more often than average risk individuals [19].

In order to detect adenomatous polyps and cancer it is recommended to use the following tests: FS every 5 years, colonoscopy every 10 years, computed tomographic colonography (CT colonography) every 5 years and doublecontrast barium enema every 5 years [19].

For detecting cancer, it is recommended the annual guaiac-based FOBT with high test sensitivity for cancer, annual fecal immunochemical test (FIT) with high test sensitivity for cancer and the stool DNA test which has a high sensitivity for cancer at an uncertain interval [19].

The guidelines of $A C G-2008$ support the division of screening tests into two main categories: cancer prevention 
tests and cancer detection tests, but recommends a preferred cancer prevention test - colonoscopy every 10 years and a preferred cancer detection test - FIT [20].

The guidelines of $A C P-2012$ recommend that average-risk individuals, aged between 50 to 70 years old, should be screened for CRC by one of the following strategies: annual high-sensitivity FIT or FOBT; colonoscopy every 10 years, flexible sigmoidoscopy every 5 years, annual high-sensitivity FIT or FOBT, high-sensitivity FIT or FOBT every 3 years plus flexible sigmoidoscopy every 5 years. However, for average-risk adults younger than 50 years old, individuals older than 75 years old or with an estimated life expectancy of less than 10 years screening for CRC is not recommended [21].

NCCN - 2013 has different guidelines for averagerisk and high-risk individuals. The recommendations for average-risk individuals are similar to the ones recommended by the Joint made of ACS, MSTF and ACR [22].

USPSTF - 2016 considers that screening for CRC should begin at the age of 50 and end at 75 years old. For individuals aged 76 to 85 years old, the choice of screening should be individualized, taking into consideration the patient's general health status and prior screening history. Furthermore, USSTF does not recommend screening for CRC for adults above 85 years old [23]. Stool-based screening test and intervals are represented by: FOBT and FIT, every year and FIT-DNA every 1 or 3 years. Direct visualization screening test and intervals are: colonoscopy, every 10 years; FS every 5 years; CT colonography every 5 years [23].

MSTF - 2017 which represents the American College of Gastroenterology (ACG), the American Gastroenterological Association (AGA), and The American Society for Gastrointestinal Endoscopy (ASGE) groups the CRC screening tests into 3 tiers based on costs, practical considerations, and performance features. The first-tier tests are annual FIT and colonoscopy every 10 years. The second-tier tests include the FIT-fecal DNA test every 3 years, FS every 5 to 10 years and CT colonography every 5 years. Capsule colonoscopy every 5 years is a third-tier test because of limited evidence and current obstacles to use [24].

ACS - 2018 reviewed its CRC screening guidelines, recommending that regular screening (with either a highsensitivity stool-based test or a structural examination, depending on patient preference and test availability) for individuals at average risk should start at 45 years old. Another recommendation refers to the fact that regular CRC screening should be performed until the age of 75 for people with a good health status and with a life expectancy of more than 10 years. Moreover, the medical provider should discuss with individuals aged between 76 and 86 whether they should continue screening or not by taking into account their personal choices, life expectancy, general health status and prior screening history; For individuals aged over 85 years old is it recommended that colorectal cancer screening should not be performed. The ACS recommendations for CRC screening are: FIT annually; high-sensitivity, FOBT annually; multitarget stool DNA test every 3 years; colonoscopy every 10 years; CT colonography every 5 years; and FS every 5 years [10].

\section{Discussion}

The majority of the guidelines recommend to start the CRC screening for average-risk individuals at age 50, based on the steep increase of CRC beginning around this age [3]. These guidelines also recommend an upper age screening threshold varying from age 70 to 75 , based on associated harms potentially exceeding benefits if screening is continued after that age [25]. The most recent guidelines of ACS (2018) recommend that adults aged 45 years and older with an average risk of CRC should undergo regular screening with either a high-sensitivity stool-based test or a structural (visual) examination, depending on the patient's preferences and test availability [10].

All the guidelines have considered FOBT (annual or biennial), FIT (annual), FS (every 5 years) and colonoscopy (every 10 years) as the most preferred screening options. However, there are discrepancies with regards to which tests should be preferred for screening. CRC screening intervals remain an area of uncertainty. When choosing the most appropriate screening intervention to improve and optimize the real-life effectiveness of CRC screening, resource availability and individual preferences should be considered as well [3].

Regular screening is considered the most effective method of detecting precancerous growths and CRC as early as possible. Cancers that are identified at an early stage can be treated more easily and lead to greater survival [17]. The benefits of adoption and promoting guidelines for screening for CRC can lead to ninety percent of all individuals with CRC at an early stage to be alive after 4 years [25].

Targeted public health programs and the involvement of the medical community can develop effective methods to communicate the importance of screening and the options of CRC screening to the public. It is a well-documented fact that awareness can boost the screening rates [26]. There is a high interest in the role of primary care providers to encourage regular screening taking into account existing guidelines due to the fact that they have a high-level of contact with the target population at risk [27]. Primary care practice is usually the first option for delivering CRC screening services through education, prescriptions for screening tests and also recommendations [28].

\section{Conclusion}

Considering the importance of CRC guidelines, the adoption of these recommendations can lead to major reductions in the CRC mortality, saving a big percentage 
of lives per year. The widespread adoption of these guidelines requires involvement from different health care institutions. Furthermore, at the national and local level, multiple targeted efforts should be made in order to expand CRC screening. The recommendations and advices of a healthcare physician are considered major determinants of patients deciding to screen and comply to the recommendations received.

\section{References}

1. Dubé C. Tackling colorectal cancer as a public health issue: what can the gastroenterologist do? Can J Gastroenterol. 2012;26:417-418.

2. Haggar FA, Boushey RP. Colorectal cancer epidemiology: incidence, mortality, survival, and risk factors. Clin Colon Rectal Surg. 2009;22:191-197.

3. Bénard F, Barkun AN, Martel M, von Renteln D. Systematic review of colorectal cancer screening guidelines for average-risk adults: Summarizing the current global recommendations. World J Gastroenterol. 2018;24:124-138.

4. Ait Ouakrim D, Pizot C, Boniol M, Malvezzi M, Boniol M, Negri E, et al. Trends in colorectal cancer mortality in Europe: retrospective analysis of the WHO mortality database. BMJ. 2015;351:h4970.

5. Trifan A, Cojocariu C, Sfarti C, Goldiş E, Seicean A, Azoicăi D, et al. Colorectal cancer in Romania: epidemiological trends. Rev Med Chir Soc Med Nat Iasi. 2006;110:533-539.

6. Favoriti P, Carbone G, Greco M, Pirozzi F, Pirozzi RE, Corcione F. Worldwide burden of colorectal cancer: a review. Updates Surg. 2016;68:7-11.

7. Arnold M, Sierra MS, Laversanne M, Soerjomataram I, Jemal A, Bray F. Global patterns and trends in colorectal cancer incidence and mortality. Gut. 2017;66:683-691.

8. Inadomi JM, Vijan S, Janz NK, Fagerlin A, Thomas JP, Lin $\mathrm{YV}$, et al. Adherence to colorectal cancer screening: a randomized clinical trial of competing strategies. Arch Intern Med. 2012; 172:575-582.

9. Hol L, de Bekker-Grob EW, van Dam L, Donkers B, Kuipers EJ, Habbema JD, et al. Preferences for colorectal cancer screening strategies: a discrete choice experiment. Br J Cancer. 2010;102:972-980.

10. Wolf AMD, Fontham ETH, Church TR, Flowers CR, Guerra $\mathrm{CE}$, LaMonte SJ, et al. Colorectal cancer screening for averagerisk adults: 2018 guideline update from the American Cancer Society. CA Cancer J Clin. 2018;68:250-281.

11. Simon K. Colorectal cancer development and advances in screening. Clin Interv Aging. 2016;11:967-976.

12. Centers for Disease Control and Prevention. CDC - What Are the Risk Factors for Colorectal Cancer? Centers for Disease Control and Prevention. 2018.

13. Siegel RL, Miller KD, Jemal A. Cancer statistics, 2018. CA Cancer J Clin. 2018;68:7-30.

14. Meester RG, Doubeni CA, Zauber AG, Goede SL, Levin
TR, Corley DA, et al. Public health impact of achieving $80 \%$ colorectal cancer screening rates in the United States by 2018 . Cancer. 2015;121:2281-2285.

15. Willyard C. Screening: Early alert. Nature. 2015;521:S4-S5.

16. Popa AE. Cancer Primary Prevention Policies Total high energy teletherapy units / million inhabitants Cancer Plans, Monitoring and Surveillance Cancer Treatment and Palliative Care. 2010.

17. Issa IA, Noureddine M. Colorectal cancer screening: An updated review of the available options. World J Gastroenterol. 2017;23:5086-5096. .

18. Kahi CJ, Imperiale TF, Juliar BE, Rex DK. Effect of screening colonoscopy on colorectal cancer incidence and mortality. Clin Gastroenterol Hepatol. 2009;7:770-775; quiz 711.

19. Levin B, Lieberman DA, McFarland B, Smith RA, Brooks $\mathrm{D}$, Andrews KS, et al. Screening and surveillance for the early detection of colorectal cancer and adenomatous polyps, 2008: a joint guideline from the American Cancer Society, the US MultiSociety Task Force on Colorectal Cancer, and the American College of Radiology. CA Cancer J Clin. 2008;58:130-160.

20. Rex DK, Johnson DA, Anderson JC, Schoenfeld PS, Burke $\mathrm{CA}$, Inadomi JM, et al. American College of Gastroenterology guidelines for colorectal cancer screening 2009 [corrected]. Am J Gastroenterol. 2009;104:739-750.

21. Qaseem A, Denberg TD, Hopkins RH Jr, Humphrey LL, Levine J, Sweet DE, et al. Screening for colorectal cancer: a guidance statement from the American College of Physicians. Ann Intern Med. 2012;156:378-386.

22. Burt RW, Cannon JA, David DS, Early DS, Ford JM, Giardiello FM, et al. Colorectal cancer screening. J Natl Compr Canc Netw. 2013;11:1538-1575.

23. US Preventive Services Task Force, Bibbins-Domingo K, Grossman DC, Curry SJ, Davidson KW, Epling JW Jr, et al. Screening for Colorectal Cancer: US Preventive Services Task Force Recommendation Statement. JAMA. 2016;315:2564-2575. 24. Rex DK, Boland CR, Dominitz JA, Giardiello FM, Johnson DA, Kaltenbach T, et al. Colorectal Cancer Screening: Recommendations for Physicians and Patients from the U.S. MultiSociety Task Force on Colorectal Cancer. Am J Gastroenterol. 2017;112:1016-1030.

25. National Cancer Institute. Cancer Stat Facts: Colorectal Cancer. 2018.

26. Doubeni CA. The impact of colorectal cancer screening on the US population: is it time to celebrate? Cancer. 2014;120:28102813.

27. Dodd N, Mansfield E, Carey M, Oldmeadow C, SansonFisher R. Have we increased our efforts to identify strategies which encourage colorectal cancer screening in primary care patients? A review of research outputs over time. Prev Med Rep. 2018;11:100-104.

28. Wang H-L, Christy SM, Skinner CS, Champion VL, Springston JK, Perkins SM, et al. Predictors of stage of adoption for colorectal cancer screening among African American primary care patients. Cancer Nurs. 2014;37:241-51. 